\title{
Redes de cooperación empresarial internacionales en el sector ambiental y cooperación triangular
}

International Business Cooperation Network and the Environmental Enterprises and Triangular Cooperation

\section{Redes de cooperação empresarial internacionais no setor ambiental e cooperação triangular}

\author{
Tania Elena González Alvarado* \\ María Antonieta Martín Granados
}

Fecha de recibido: 3 de enero de 2017

Fecha de aprobado: 4 de agosto de 2017

Doi: http://dx.doi.org/10.12804/revistas.urosario.edu.co/empresa/a.5307

Para citar: González Alvarado, T. E., \&, Martín Granados, V. M. A. (2018). Redes de cooperación empresarial internacionales en el sector ambiental y cooperación triangular. Universidad \& Empresa, 20(35), 51 1-77. Doi: http://dx.doi.org/10.12804/revistas.urosario.edu.co/empresa/a.5307

\section{RESUMEN}

El objetivo de este artículo es analizar el comportamiento de las empresas del sector ambiental en la red de cooperación empresarial internacional. A fin de alcanzarlo se analizaron 130 empresas del sector ambiental de diferentes países que participaron en encuentros

* Doctorado en Economía Internacional y Desarrollo de la Universidad Complutense de Madrid. Profesora-Investigadora Titular tiempo completo en la Universidad de Guadalajara, México, Departamento Mercadotecnia y Negocios Internacionales. Correo electrónico: taniaelena_2003@ yahoo.es

** Doctorado en Ciencias de la Administración de la Universidad Nacional Autónoma de México. MBA por la Universidad de Tulane (Estados Unidos). Correo electrónico: mmartin@correo.fca. unam.mx 
empresariales Al-Invest. El principal resultado es que al vincularse las empresas entre diferentes regiones buscan, principalmente, acuerdos de representación (32,58\%) y búsqueda de empresas productoras (31,82\%). En igualdad de importancia (25\%) se identificaron tres fines adicionales: transferencia de conocimiento, ofertas de productos y comercialización. La conclusión es que los vínculos entre las empresas estudiadas reflejan la cooperación Norte-Sur-Sur, viéndose esta última facilitada por la cooperación triangular. Palabras clave: PYME, cooperación para el desarrollo internacional, vínculo multiempresa.

\section{ABSTRACT}

The aim of this paper is to analyze the environmental enterprises behavior in the international business cooperation network. 130 environmental companies that had participated in business meetings Al-Invest from different countries were analyzed. The main finding was that the environmental enterprise seeks for representation agreements $(32.58 \%)$ and production companies $(31.82 \%)$. Besides, other findings were that knowledge transfer, product offerings and marketing achieve the same percentage $(25 \%)$. The established conclusion was that cooperation between the environmental companies reflect the NorthSouth-South cooperation, with the latter facilitated by triangular cooperation.

Keywords: SMEs, international cooperation and development, multi-enterprise tie.

\section{RESUMO}

O objetivo deste artigo é analisar o comportamento das empresas do setor ambiental a rede de cooperação empresarial internacional. Com o fim de alcançá-lo se analisaram 130 empresas do setor ambiental de diferentes países que participaram em encontros empresariais Al-Invest. O principal resultado é que ao vincular-se as empresas entre diferentes regiões procuram, principalmente, acordos de representação (32.58\%) e busca de empresas produtoras (31.82\%). Em igualdade de importância (25\%) se identificaram três fins adicionais: Transferência de conhecimento, Ofertas de produtos e Comercialização. A conclusão é que os vínculos entre as empresas estudadas refletem a cooperação Norte-Sul-Sul, resultando esta última facilitada pela cooperação triangular.

Palavras-chave: MPE, cooperação para o desenvolvimento, vínculo multiempresa. 


\section{INTRODUCCIÓN}

La teoría económica se ha concentrado en el impacto ambiental de cara a la construcción de indicadores e instrumentos para disminuir el efecto negativo de las actividades de transformación y extracción (Canter, et al.,1998; Leonard, et al.,1987; Porter, et al., 1991; Martínez, et al., 1995; Castro, 2002; Falconí \& Burbano, 2004; Achkar, 2005; Aguilera, et al. 1995; Buenaventura, et al., 2006). Sin embargo, existe un gran vacío en términos de estudios sobre las empresas que operan en el sector ambiental. Cuando se aborda el fenómeno desde la perspectiva empresarial, este primero queda encajonado en la Responsabilidad Social y en iniciativas para la reconversión de la empresa con menor impacto negativo al ambiente (Aray, et al., 2002; Panwar \& Hansen, 2008; Sandrea \& Boscán, 2010; Vargas, et al., 2011; Bercovich \& Chidiak, 1994).

Difícilmente se acota el objeto de estudio hacia las empresas dedicadas a ofrecer servicios o bienes ambientales (Villareal, 2006). De hecho, algunos trabajos se refieren a servicios ambientales como la valoración y la comercialización de servicios de la naturaleza que antes se hallaban excluidos de un intercambio o posible mercadeo (Pascual \& Corbera, 2011; Penna \& Cristeche, 2008; Fonseca,
2006; Campos, et al., 2006; TorresCarral, 2006; Lomas, et al., 2005; Castiblanco, 2003; Campos-Arce, et al., 2001), mientras que en el presente escrito se entiende por servicio ambiental a la consultoría, asesoría y ayuda técnica que una empresa puede ofrecer en el mercado en materia ambiental.

Se espera que las empresas que ofrecen productos y servicios para la mejora en el ambiente contribuyan al desarrollo sostenible. Han pasado casi diez años en que Schaper (2007) planteara la importancia del sector ambiental para América Latina. Después de una década este sector continúa diluyendo sus actividades entre los otros sectores económicos. Aún no se cuenta con una base de datos integrada que permita dar cuenta de los avances empresariales dentro del sector, ya que las bases de datos que se han conformado se enfocan directamente en monitorear los cambios en el ambiente y las modificaciones a la existencia de los recursos naturales.

Por tanto, abordar a las empresas de dicho sector conlleva a la formación de bases propias, con la limitante principal de que no existe un puente claro entre estas bases con el comportamiento de la inversión extranjera directa, las importaciones y exportaciones, y la contribución del sector al 
Producto Interno Bruto. Tampoco a la generación de puestos de empleos con las ventajas competitivas alcanzadas por estas empresas. También es un reto para agendas posteriores de investigación generar bases oportunas, congruentes e integradas para el análisis de la competitividad del sector ambiental.

\section{REVISIÓN DE LA LITERATURA}

\subsection{Limitaciones para} medir la competitividad del sector ambiental a través de indicadores económicos

Según Schaper (2007), América Latina y el Caribe es un mercado en expansión para la industria de los bienes y servicios ambientales, principalmente por sus carencias en materia de infraestructura ambiental, asociadas al crecimiento demográfico y la alta densidad urbana que caracterizan la región. De acuerdo con este autor, es un mercado que comienza a responder a los nuevos marcos regulatorios y a una mayor preferencia social a favor de la protección ambiental; este hecho ha sido más pronunciado en los países con una especialización exportadora centrada en industrias ambientalmente sensibles, que enfrentan crecientes exigencias ambientales en los mercados internacionales (Schaper, 2007).
Por ser un mercado emergente y de rápida expansión ha sido propicio para la Inversión Extranjera Directa, y en especial para la inversión europea, que ha enfatizado su apuesta por el sector energético latinoamericano. Dicho sector es clave para las actividades económicas globales y está íntimamente relacionado con el ambiental; sin embargo, el análisis del sector ambiental tras la apertura comercial de la región latinoamericana resulta complicado porque este sector no ha sido definido de forma homogénea, es decir, no existen indicadores consistentes y claros a través del tiempo que puedan reflejar las actividades de las empresas y el comportamiento de la inversión extranjera directa en este rubro, lo que obliga a generar bases de datos propias para cada proyecto de estudio. Esta situación ya ha sido puesta a debate por otros investigadores, que se incluyen en el presente trabajo. Uno de esos trabajos corresponde a Barton (1998) que denomina al sector como "ecoindustria", haciendo con ello alusión a los problemas ecológicos que han instado al surgimiento de actividades económicas proambientales.

Barton (1998) plantea que la ecoindustria y las tecnologías menos contaminantes deberían entenderse como industrias y no como conjuntos de productos y servicios de 
valor ecológico incuestionable. Asimismo, analiza la medida en que estas industrias revelan lagunas de información y tecnología en materia de gestión ambiental. Estas lagunas podrían ser útiles para ayudar los gestores ambientales de Latinoamérica, pero también podrían generar una condición de dependencia en esta materia (Barton, 1998).

La crítica expuesta por Barton (1998) es importante, debido a que un planteamiento alterno para las actividades económicas podría reducir la forma tradicional de contaminación, pero podría generar nuevas formas de contaminación. Estas nuevas formas, no necesariamente serían menores pero sí diferentes. De ahí la importancia que toman las empresas dedicadas a medir el daño ambiental.

Existe la posibilidad de que se contamine más, pero que sólo a largo plazo se desarrollen medios para detectar el impacto ambiental negativo; tal vez en el momento de surgir la innovación no existan dichos medios, por no contar con mayor evidencia sobre el impacto negativo porque la prioridad es la alternancia. Dicha situación se abre a una realidad, toda vez que los esfuerzos en i+D en el sector están encaminados a la sustitución y a la construcción de alternativas enfocadas en el proceso de transformación económica y no directamente encaminadas en la comprensión del ambiente y su conservación.

El surgimiento de las ecoindustrias, particularmente de las dedicadas a la producción de bienes y servicios destinados a reducir la contaminación (principalmente tecnologías de última etapa) fue un fenómeno universal en los ochenta del siglo pasado. Esto fue una respuesta a regulaciones más estrictas. Los Estados Unidos, Japón y Alemania fueron los pioneros en el desarrollo de la ecoindustria, pero, según Barton (1998), es relevante el papel de la eco-industria en el desarrollo industrial latinoamericano del siglo XXI, tanto en términos del suministro de bienes y servicios como del mejoramiento de la calidad ambiental de la región (Barton, 1998).

La Organización para la Cooperación y el Desarrollo Económicos (OCDE, 1999) define a la industria de bienes y servicios ambientales como aquella que produce bienes y servicios para medir, prevenir, limitar, minimizar o corregir el daño ambiental al agua, aire y suelo, así como los problemas relacionados a residuos, ruidos y ecosistemas. Esto incluye tecnologías más limpias y productos y servicios que reducen el riesgo ambiental y minimizan la contaminación y el uso 
de los recursos. Esta definición es más amplia que la de Barton (1998) e incluye a las empresas u organizaciones que desarrollan y aplican herramientas que permiten medir el daño ambiental.

De acuerdo con la Agencia Alemana para la Cooperación Técnica (German Agency for Technical Cooperation, 2005), al analizar las empresas ambientales de Colombia, Chile y México, se observa que, en general, no operan bajo la definición otorgada por la OCDE (1999), pues las empresas tienden a autoclasificarse dentro o fuera de este mercado. Los bienes y servicios ambientales no se encuentran claramente especificados en los registros de importaciones y exportaciones de los países, debido a que no existen códigos armonizados para una amplia gama de bienes y servicios ambientales. De hecho, existen diferentes clasificaciones en el mundo desarrollado que se encuentran en proceso de unificación, lo que dificulta cualquier análisis y crea controversias. (German Agency for Technical Cooperation, 2005)

El estudio de la German Agency for Technical Cooperation (2005) se enmarca en la línea de identificación de oportunidades en el sector ambiental de América Latina y el Caribe. El objetivo de estudio fue contribuir al desarrollo de un sector económico que pueda asegurar los beneficios de las oportunidades de un mercado de bienes y servicios ambientales en expansión, y satisfacer una demanda creciente en el sector de protección ambiental, particularmente a través de alianzas o joint ventures.

En dicho estudio (German Agency for Technical Cooperation, 2005) se analizaron las experiencias de colaboración o alianzas entre empresas de menor tamaño en el nivel internacional, particularmente relevantes y exitosas. También se realizó una identificación de industrias proveedoras de bienes y servicios ambientales internacionales más idóneas para formar alianzas (partnerships o joint ventures) con pequeñas y medianas empresas latinoamericanas. Las alianzas público-privadas que la Agencia Alemana para la Cooperación Técnica analizó fueron Programa Alianzas de Negocio UNIDO, ECOPROFIT, Alianzas Ambientales Chile-Suecia, Programa PPP de GTZ, Proyecto de Transferencia de Tecnología Quebec-Chile y más de 190 alianzas privadas-privadas entre empresas de México, Chile y Colombia. El estudio identificó que los esquemas de alianzas público-privadas más exitosos, de acuerdo con el logro de objetivos, cumplimiento de productos comprometidos y cumplimiento de metas, a través de indicadores 
cualitativos y/o cuantitativos fueron el Programa Alianzas de Negocio UNIDO y ECOPROFIT. En general, esta agencia observó falta de objetivos claros y de una evaluación de resultados económicos, tanto en el nivel de las empresas como del país y la organización promotora de la alianza. La consideración de las alianzas como un proceso en el tiempo, en el que interactúan diferentes actores que obtienen logros y aprenden de fracasos se pierde, es decir, el proceso de aprendizaje, construcción de redes y generación del conocimiento no es adecuadamente valorado ni retenido por las organizaciones (German Agency for Technical Cooperation, 2005).

Los resultados alcanzados muestran que el sector ambiental ha surgido de una necesidad multilateral; debido a su carácter prescriptivo, las acciones han sido desarticuladas y con menor impacto al esperado. Mientras que los agentes económicos involucrados en el nivel empresa no consideren con el mismo nivel de importancia que los gobiernos las medidas tomadas en pro del ambiente, difícilmente las actividades económicas alcanzarán su propia sustentabilidad. Apenas se desmantele algún programa o se elimine determinado estímulo fiscal, estas actividades desaparecerán con mayor velocidad con la que fueron creadas.
El surgimiento del sector ambiental ha sido de "arriba hacia abajo", lo que tiene como consecuencia la discrepancia, la cual se traduce en una barrera para el estudio del sector ambiental; esto último se observa en las leyes ambientales, que suelen ser diferentes en cada región de Latinoamérica. Todos los países de la región tienen políticas ambientales, pero no suelen coordinarse con las medidas de promoción de la inversión (CEPAL, 2015). Aun así, casi dos tercios de los organismos de promoción de la inversión afirman que tienen en cuenta el impacto ambiental de la Inversión Extranjera Directa y, a pesar de su limitado margen de maniobra, muchos cuentan con programas para atraer inversión extranjera directa según criterios ecológicos, con un fuerte énfasis en las energías renovables (CEPAL, 2015).

La Comisión para América Latina y el Caribe (CEPAL, 2015) sugiere a los gobiernos que garanticen la consistencia entre las políticas de promoción de la IED y otras políticas con efectos sobre el medioambiente, como la energética, transporte, industria y desarrollo urbano. La CEPAL (2016) insta a la construcción de un espacio integrado y con reglas comunes para promover los encadenamientos productivos, aumentar la resiliencia del comercio 
intrarregional y favorecer la diversificación productiva y exportadora. Lo anterior indica oportunidades de negocio internacionales para las empresas de menor tamaño, particularmente para aquellas que operan en regiones de renta baja, en las que la pobreza y la exclusión son la regla. Esto último abre la oportunidad de crear empresas en el sector ambiental con un elevado impacto en el desarrollo económico local, que además estén colaborando con más de un Objetivo del Desarrollo Sostenible, debido a que se encuentran en un sector clave para alcanzar dichos objetivos. También exige estudiar el comportamiento de las empresas ya existentes en el sector y buscar mecanismos que permitan a las empresas consolidarse en él bajo estrategias de "abajo hacia arriba" con mayor impacto local y sustentabilidad para los negocios y no solo para el medio ambiente. Finalmente, los programas multilaterales y públicos están abriendo mercado, lo que genera y con ello nuevas oportunidades para las empresas, especialmente para las de menor tamaño.

\subsection{Oportunidades para las empresas de menor tamaño}

Entre las acciones que favorecen a las empresas del sector ambiental, se encuentran las registradas por el
Banco Mundial (2014) en el contexto del programa de desarrollo limpio; este se enfoca en ayudar a los países a encontrar sendas de desarrollo que admitan un bajo nivel de emisiones, a través de:

- El fomento de un intercambio Sur-Sur sobre las mejores prácticas para la gestión de la contaminación.

- La ampliación del uso de cocinas más limpias que contribuyan a disminuir la polución en los espacios cerrados.

- El apoyo a los países en los asuntos relativos a la limpieza de los ríos y la contaminación histórica.

- El mejoramiento de la eficiencia energética, fomentando el uso de energías renovables, encontrando soluciones agrícolas a favor de la adaptación al cambio climático y construyendo ciudades más limpias y con niveles más bajos de carbono.

Analizar la forma en que este tipo de acciones deriva en oportunidades para los negocios en el sector ambiental exige perfilar, agrupary clasificar a las empresas implicadas. Buil, Fraj y Matute (2005) identificaron estudios que agrupan a las empresas en términos ambientales: los factores de presión medioambiental sobre el comportamiento empresarial 
(Del Brío \& Junquera, 2001; Banerjee, 2002); la preocupación y actitud medioambiental (Fundación Entorno, 2003); la percepción sobre los efectos de los Sistemas de Gestión Medioambiental en su posición competitiva (Giménez, et al., 2002), y la valoración que realizan sobre las ventajas e inconvenientes por la implantación de estrategias medioambientales (Ruzo, et al., 2005). Tanto el enfoque de Buil, Fraj y Matute (2005) como el de los demás autores se refiere a empresas que no operan directamente en el sector ambiental, pero que en cierto grado se preocupan por aspectos ambientales. En este sentido, las empresas de menor tamaño difícilmente implementan sistemas para la protección del medioambiente (Ortiz et al. 2013); a no ser que operen directamente en el sector ofreciendo consultoría o productos vinculados a la protección ambiental.

Esto último indica que en las grandes empresas es viable encontrar un área sobre impacto ambiental que sea adicional para las actividades estratégicas de la empresa, mientras que en las empresas de menor tamaño esto difícilmente ocurrirá. Es posible que en el caso de la comercialización y consultoría se encuentre un esquema contrario, sea un mayor número de empresas de menor tamaño dedicadas a la oferta tanto de consultoría como de productos que disminuyan el impacto negativo en el ambiente. Por otra parte, debido a la naturaleza de la problemática, la mayor inversión en Investigación y Desarrollo en materia ambiental está representada por organismos multilaterales y agencias de gobierno, antes que por las propias empresas.

A través de diversos estudios, se ha demostrado que los sectores químico y manufacturero presentan una mayor orientación a la estrategia medioambiental, puesto que tienen que hacer frente a una alta regulación sobre esta materia. En cambio, los servicios son los que menor orientación presentan porque la legislación medioambiental que soportan es mucho más flexible (Banerjee, 2002; Buil et al. 2005); además, los grupos empresariales pueden llegar a agruparse para presionar a los gobiernos de forma que disminuyan las presiones ambientales (Camacho, 2016).

Debido a la diversidad de contextos que conducen a que las empresas operen en el sector ambiental, así como a la ausencia de bases de datos que permitan identificarlas para su estudio, en el caso del presente proyecto se toma como empresas del sector ambiental a aquellas que al postularse para los apoyos del 
programa europeo Al-Invest declararon operar en este sector; en otras palabras, son empresas que declararon ofrecer productos y servicios en el mercado para reducir el impacto ambiental. El presente proyecto de investigación pretende analizar los fines por los que estas empresas establecen vínculos con agentes económicos de otras regiones, insertándose de esta forma en redes de cooperación empresarial internacionales que facilitan la entrada a mercados extranjeros.

\subsection{Red de cooperación empresarial internacional}

Los vínculos y tramos construidos por las empresas (Lafer, 1973; Abdenur \& De Souza, 2013; Bueno \& Saraví, 1997; Tabares, et al., 2014; Yoguel \& Bercovich, 1994; González, 2007) en la búsqueda de una mayor competitividad a través de la expansión del mercado y la reducción de costos (Liendo \& Martínez, 2001; Donovan, et al., 2004; Tkachuk, 2004; Kulfas, 2009; Velásquez, 2004 ) han generado redes de cooperación empresariales internacionales con la posibilidad de conectarse o establecer relaciones con otras redes. Esto ocurre cuando los elementos de una de ellas establecen relaciones con los elementos de otra, con lo que se crean entre ellas redes cada vez más grandes y complejas que permi- tirán, mientras mantengan la misma lógica para el establecimiento de los vínculos, que los elementos se conecten sin importar la región a la que pertenezcan (González, 2003).

Las barreras culturales, geográficas y legales pasan a ser casi nulas al interactuar los agentes de diferentes regiones bajo el mecanismo de cooperación (Asia, Europa, Europa del Este o América Latina). Esto no significa que dichas barreras desaparezcan; más bien, implica que los agentes que han decidido cooperar se esfuerzan por salvarlas en un intento por vincularse y alcanzar sinergias que se traduzcan en nuevos recursos, mejores capacidades $y$, finalmente, en ventajas competitivas en constante transformación (figura 1). El tamaño y forma de la red es difícil de identificar, aunque los estudios de Axelrod (1984) permiten proponer una topología; sin embargo, esta topología se basa en principios lógicos y la teoría de juegos, considerándose que la realidad es más compleja (González, 2006). Tómese en cuenta que se está analizando una red que opera internacionalmente, e incluso en algunos casos, como el automotriz, opera globalmente; lo anterior vuelve complejo su modelado, tanto por su dinámica como por su extensión, ya que mientras unos vínculos se crean otros se rompen. 
La lógica de este sistema de cooperación en red se caracteriza por los siguientes puntos: 1) el mecanismo de cooperación es el factor vinculante; 2) los agentes han aprendido a ver el fracaso y el oportunismo como parte del aprendizaje; 3) los involucrados están convencidos de que cooperando obtendrán mayores beneficios que compitiendo; 4) cuentan con un proceso de negociación permanente; 5) consideran a la contraparte como un medio para el alcance de sus objetivos y la generación de sinergias.

Dentro de esta red, las principales actividades que la fundamentan no se localizan en los encuentros empresariales ni en los organismos públicos, más bien, son las tecnologías de información y comunicación las que han permitido actividades clave para su sostenimiento. Tal es el caso de las videoconferencias que permiten un sistema de comunicación permanente entre las empresas vinculantes; dicho sistema de comunicación a través de la tecnología permite a los colaboradores con- tinuar negociando, transformando así las circunstancias que favorecen el oportunismo en momentos para nuevas negociaciones que fortalecen los vínculos y permiten alcanzar nuevas sinergias. La negociación se extiende más allá de un paternariado o encuentro empresarial, siendo un elemento fundamental para la existencia del vínculo y sus resultados (figura 1).

Otra gran ventaja que ofrecen estas tecnologías es la oportunidad de administrar en forma conjunta los recursos, su aplicación y los resultados a través de sistemas informáticos y la minería de datos (figura 1). Lamentablemente, cuando se aborda la tecnología en los vínculos de cooperación internacionales, se suele encajonar el fenómeno en el uso de las redes sociales y las páginas web, cuando estos dos elementos se quedan limitados para los vínculos de cooperación, dado que van más encaminados a mantener y hacer crecer la demanda antes que a consolidar los vínculos entre los empresarios de diferentes regiones. 
Figura 1. Generación de ventaja competitiva dentro del vínculo empresarial bajo el mecanismo de cooperación

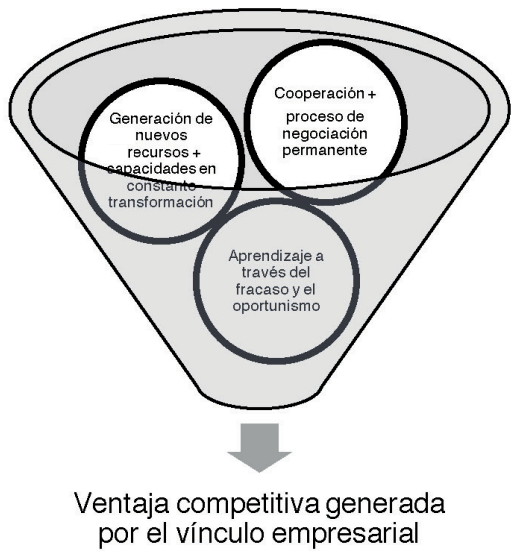

Fuente: elaboración propia.

Una de las razones por las cuales existe esta limitación se debe al carácter abierto de estas dos últimas herramientas, que han sido diseñadas para la exposición pública $\mathrm{y}$, por tanto, para la construcción de una imagen ante el mercado. Por supuesto que pueden influir de forma indirecta en la construcción de los vínculos, nadie estaría interesado en vincularse a una empresa con mala reputación, pero no son clave para la profundización de la relación en la que la confianza va acompañada de la confidencialidad y en la que los conflictos se analizan y resuelven a puertas cerradas, como sí sucede con las tecnologías cerradas.

Las tecnologías de información y comunicación han dispuesto la in- fraestructura necesaria que abre la posibilidad de que estas redes se sostengan por sí mismas (Cervilla \& Lorenzo,1999; Hernández, 2001; Tsuji, et al., 2005; Alderete, 2007; Fernández \& Narváez, 2011). Por otra parte, programas de apoyo internacionales, como Al-Invest, el libre cambio y las cadenas globales de valor, fueron factores que desencadenaron estos entramados internacionales protagonizados por empresas de menor tamaño (Liendo \& Martínez, 2001; García \& Moreno, 2007; Perego \& Marteau, 2007; Luna, 2009; Capó-Vicedo, et al., 2009; Ferraro \& Stumpo, 2010; Pla-Barber \& Escribá, 2010; Moncayo, 2010; Fernández \& Revilla, 2010; Albizu, et al., 2011; Fernández-Jardón, 2012). 
Figura 2. Entorno de cooperación internacional favorable para los vínculos entre empresas

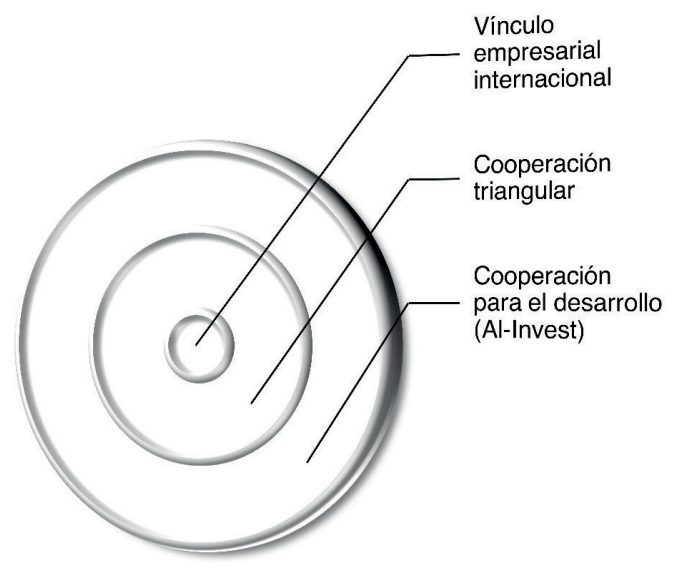

Fuente: elaboración propia.

Las empresas que operan en el sector ambiental, sin importar su tamaño, han encontrado atractiva la internacionalización a través de las redes de cooperación empresarial internacionales en el marco de la Cooperación para el Desarrollo (Figura 2). Esto les permite incursionar en nuevas regiones con oficinas de representación, obtener alianzas público- privadas a través de una empresa ya establecida en el país destino y educar al mercado potencial en el uso de los bienes y servicios que ofrece, todo ello con menor riesgo que actuando de forma independiente. Esto conduce a cuestionarse ¿qué tan antiguas son estas empresas?, ¿cuál es el país de origen?, ¿cuál es el país destino?, ¿qué buscan en un vínculo de cooperación internacional?

\section{METODOLOGÍA}

En cuanto a la base de datos sobre el sector ambiental, se inició con las empresas que participaron en una o más ferias empresariales internacionales. Estos eventos fueron organizados por el Eurocentro Nafin en el periodo 2002-2009 (16 encuentros con un total de 2724 empresas participantes). Se eliminaron a las empresas que habían desaparecido y se trabajó sólo con aquellas que cubrían los siguientes requisitos: contar con una página web, mantener vínculos con empresas de otras regiones y estár incluidas en diferentes directorios. Se trabajó con los directorios de los eventos organizados por el Eurocentro Nafin a partir del 2002, porque se requería mayor 
distancia entre la situación actual de las empresas y los encuentros, a fin de poder analizar en retrospectiva el comportamiento de los vínculos entre empresas y otros agentes locales. De esta forma, se obtuvo un grupo de estudio conformado por $130 \mathrm{em}$ presas del sector ambiental (tabla 1). Los resultados se alcanzaron con base en la información proporcionada por el Eurocentro, las búsquedas de información a través de la web; entrevistas, visitas de campo y llamadas telefónicas.

Tabla 1. Participación de las empresas del sector ambiental por país en que se encuentran establecidas

\begin{tabular}{|c|c|c|}
\hline Países & Cantidad & Porcentaje \\
\hline Alemania & 9 & $6,9 \%$ \\
\hline Argentina & 3 & $2,3 \%$ \\
\hline Brasil & 1 & $0,8 \%$ \\
\hline Chile & 3 & $2,3 \%$ \\
\hline Colombia & 5 & $3,8 \%$ \\
\hline Costa Rica & 1 & $0,8 \%$ \\
\hline España & 10 & $7,7 \%$ \\
\hline Francia & 5 & $3,8 \%$ \\
\hline Honduras & 1 & $0,8 \%$ \\
\hline Italia & 5 & $3,8 \%$ \\
\hline México & 86 & $66,2 \%$ \\
\hline Países Bajos & 1 & $0,8 \%$ \\
\hline Total & 130 & \\
\hline
\end{tabular}

Fuente: elaboración propia con base en los resultados alcanzados en el proyecto Generación de valor y cooperación internacional en las empresas de menor tamaño de Iberoamérica UDG-CA-484.
Flyvbjerg (2006) señala que, cuando el objetivo es lograr la mayor cantidad posible de información sobre un determinado problema o fenómeno, un caso representativo o una muestra aleatoria puede no ser la estrategia más apropiada. Ello se debe a que el típico caso o el caso medio suele no proporcionar la mejor ni la mayor información. Los casos atípicos o extremos suelen revelar más información porque activan más actores y más mecanismos básicos en la situación que se estudia. Además, desde una perspectiva orientada tanto a la comprensión como a la acción, suele ser más importante clarificar las causas profundas de un determinado problema y sus consecuencias que describir los síntomas del problema y la frecuencia con la que estos ocurren. Las muestras aleatorias que acentúan la representatividad raramente podrán producir este tipo de conocimiento, ya que es más apropiado seleccionar algunos casos por su validez (Flyvbjerg, 2006); por esta razón, es que dentro del proyecto se profundiza en el estudio de caso, aunque esto conduce a obtener resultados con mayor lentitud y costo.

\section{RESULTADOS DE INVESTIGACIÓN Y DISCUSIÓN}

De acuerdo con el origen de la empresa, el país con mayor representación en los encuentros fue México 
(66,2 \%). Una conclusión apresurada sería que México, en comparación con otras regiones, se ha enfocado más en el sector ambiental; sin embargo, un análisis más detallado a través de las visitas de campo a las empresas y la presencia como observadores en algunos encuentros empresariales, ayudan a considerar la posibilidad de que estas empresas creadas en México corresponden a capitales extranjeros. En su mayoría, son empresas mexicanas muy pequeñas que forman parte de un corporativo multinacional, cuyo origen está en un país de renta alta. Por tanto, para un mejor análisis es conveniente quitar este grupo de empresas mexicanas y continuar el análisis sobre los otros porcentajes. Bajo esta segunda óptica, se identifica que España (10 empresas) y Alemania (9 empresas) son los países con mayor participación en el sector.

La cercanía económica e histórica con América Latina, y particularmente con México, permite explicar la existencia de empresas españolas en todos los sectores propuestos en el marco de Al-Invest. En el caso de la participación de Alemania, y los demás países tanto europeos como latinoamericanos, es posible incluir para el análisis a la cooperación triangular. Esta consideración es por tratarse del sector ambiental como eje de estudio en el marco de
Al-Invest, siendo este último un programa derivado de la cooperación para el desarrollo que ofrece la Unión Europea hacia América Latina.

En los últimos años, la cooperación triangular se ha convertido en un novedoso planteamiento que se establece entre actores de tres países: un oferente de cooperación o socio donante, un país de renta media (PRM) que actuará igualmente como socio oferente de cooperación, y un socio receptor de un país de menor nivel de desarrollo relativo. (Gómez, Ayllón \& Albarrán, 2011).

Un estudio de la Organización para la Cooperación y el Desarrollo Económicos identificó que al menos dieciséis países (Canadá, Francia, Dinamarca, Bélgica, España, Italia, Noruega, Suecia, Estados Unidos y Gran Bretaña, entre otros) estaban activos, participaban en acciones de cooperación triangular y su apoyo no se concentraba en regiones específicas. (Gómez, Ayllón \& Albarrán, 2011). Este tipo de cooperación ha sido propicia para el sector ambiental en el marco de los Objetivos del Desarrollo Sostenible, y ha sido aprovechada por Alemania en América Latina.

El Ministerio Federal de Cooperación Económica y Desarrollo de Alemania (BMZ, por sus siglas en 
alemán) se ha fijado como objetivo aprovechar en mayor medida las múltiples opciones de aplicación de la cooperación triangular para el desarrollo. Entre los socios más importantes se encuentran Brasil, México y Chile. En cuanto a México, las actividades se concentran en la transferencia de experiencias de la cooperación bilateral mexicano-alemana para el desarrollo. La idea es contribuir, por un lado, al desarrollo de otros países, y por otro, apoyar el rol de México como donante en América Latina y el aprovechamiento de capacidades existentes, por ejemplo, con Bolivia en el área de gestión de aguas residuales (BMZ, 2013). Este ejemplo muestra la relación Alemania-México-Bolivia, en el que se involucran a empresas del sector ambiental para la gestión de aguas residuales.

Esto último permitiría justificar que el $86 \%$ de las empresas de la base de datos están establecidas en México, a pesar de que no es un país líder en el sector ambiental; en este orden de ideas, el país puede ser una plataforma de la inversión de terceros países que pretenden operar en el sector ambiental latinoamericano, no necesariamente limitándose al mercado mexicano, sino expandiéndose sobre el continente.

Con base en la antigüedad de las empresas, se identifican ocho casos con casi un siglo de existencia. Estas empresas no operan desde su origen en el sector ambiental, sin embargo, sí lo hacen en sectores que requieren de una mayor adaptación de la empresa hacia un mejor manejo (explotación, producción y distribución) de fuentes de energía y de contaminantes (limpio, re-uso y re-ciclo). Esto también está en congruencia con la cooperación triangular. Tal como se muestra en la la tabla 2 , la mayor parte de las empresas del grupo de estudio se crean en paralelo a los Objetivos del Milenio y son tan jóvenes como el sector ambiental. Estas empresas estarían incluidas en las dos primeras columnas de la tabla 2.

Tabla 2. Antigüedad de la empresa del sector ambiental por país

\begin{tabular}{|c|c|c|c|c|c|c|c|}
\hline \multirow{2}{*}{ Países } & \multicolumn{6}{|c|}{ Años } & \multirow{2}{*}{ Total } \\
\cline { 2 - 8 } & $0-22$ & $23-42$ & $43-62$ & $63-82$ & $83-102$ & ND & \\
\hline Alemania & 5 & 3 & 0 & 0 & 0 & 1 & 9 \\
\hline Argentina & 2 & 0 & 0 & 1 & 0 & 0 & 3 \\
\hline
\end{tabular}




\begin{tabular}{|c|c|c|c|c|c|c|c|}
\hline \multirow{2}{*}{ Países } & \multicolumn{6}{|c|}{ Años } & \multirow{2}{*}{ Total } \\
\hline & $0-22$ & $23-42$ & $43-62$ & $63-82$ & $83-102$ & ND & \\
\hline Brasil & 0 & 0 & 0 & 0 & 1 & 0 & 1 \\
\hline Chile & 2 & 0 & 1 & 0 & 0 & 0 & 3 \\
\hline Colombia & 0 & 0 & 1 & 0 & 4 & 0 & 5 \\
\hline Costa Rica & 0 & 1 & 0 & 0 & 0 & 0 & 1 \\
\hline España & 5 & 3 & 2 & 0 & 0 & 0 & 10 \\
\hline Francia & 0 & 1 & 0 & 3 & 0 & 1 & 5 \\
\hline Honduras & 0 & 0 & 0 & 0 & 0 & 1 & 1 \\
\hline Italia & 0 & 3 & 1 & 0 & 1 & 0 & 5 \\
\hline México & 40 & 20 & 2 & 2 & 1 & 21 & 86 \\
\hline Países Bajos & 0 & 0 & 0 & 0 & 1 & 0 & 1 \\
\hline Total & 54 & 31 & 7 & 6 & 8 & 24 & 130 \\
\hline
\end{tabular}

Fuente: elaboración propia con base en los resultados alcanzados en el proyecto Generación de valor y cooperación internacional en las empresas de menor tamaño de Iberoamérica UDG-CA-484.

De acuerdo con lo que buscan las empresas del sector al vincularse internacionalmente bajo el mecanismo de cooperación, la tabla 3 muestra la finalidad de la promoción de estas empresas en los encuentros Al-Invest. Nuevamente, se encuentra coincidencia de estos fines con la cooperación triangular. Particularmente, con los objetivos de la cooperación triangular alemana para el desarrollo: 1) mayor eficacia de las medidas de política de desarrollo en los países beneficiarios, gracias al uso complementario y la acumulación de conocimientos, experiencias y recursos financieros provenientes de los países emergentes y de Alemania; 2) generación de alianzas para el desarrollo en el nivel mundial, dirigidas a lograr un desarrollo sostenible; 3) concepción conjunta de metas globales de desarrollo y fomento del intercambio de lecciones aprendidas y experiencias sobre los mecanismos de acción y los principios de la cooperación; 4) reproducción y divulgación de experiencias conjuntas de la cooperación bilateral para el desarrollo con socios globales en otros países en desarrollo; 5) fomento de la cooperación SurSur, desarrollo regional, diálogo e interconexión en redes (BMZ, 2013). 
Tabla 3. Tipo de vinculación promovida por la empresa durante el encuentro

\begin{tabular}{|l|c|c|}
\hline \multicolumn{1}{|c|}{ Finalidad } & Frecuencia & Porcentaje \\
\hline Equipamiento & 6 & 4,55 \\
\hline Proyectos llave en mano & 14 & 10,61 \\
\hline Acuerdos de representación & 43 & 32,58 \\
\hline Ofertas de productos & 33 & 25 \\
\hline Búsqueda de empresas productoras & 42 & 31,82 \\
\hline Distribución & 24 & 18,18 \\
\hline Importación & 3 & 2,27 \\
\hline Comercialización & 33 & 25 \\
\hline Concesiones & 2 & 1,52 \\
\hline Inversionistas & 5 & 3,79 \\
\hline Coinversión & 4 & 3,03 \\
\hline Socios & 10 & 7,58 \\
\hline Alianzas & 6 & 4,55 \\
\hline Franquicias & 4 & 3,03 \\
\hline Subcontratación & 6 & 4,55 \\
\hline Joint Venture & 2 & 1,52 \\
\hline Transferencia del conocimiento & 33 & 25 \\
\hline
\end{tabular}

Fuente: elaboración propia con base en los resultados alcanzados en el proyecto Generación de valor y cooperación internacional en las empresas de menor tamaño de Iberoamérica, UDG-CA-484.

En los resultados de la presente investigación se identifican los dos fines más importantes al vincularse: acuerdos de representación (32,58 \%) y búsqueda de empresas productoras $(31,82 \%)$. En igualdad de importancia $(25 \%)$ se identificaron tres fines: transferencia de conocimiento, ofertas de productos y comercialización. Los acuerdos de representación, la búsqueda de empresas productoras y la trasferencia de conocimiento orientan las actividades del sector ambiental dentro de la red internacional creada por Al-Invest hacia el uso complementario y la acumulación de conocimientos, experiencias y recursos financieros entre los países participantes, el intercambio de experiencias y lecciones aprendidas de los mecanismos de acción, los principios de cooperación, y la reproducción y divulgación de experiencias conjuntas e interconexión en redes. 
Es posible que la experiencia de las empresas en el sector sea la que influya al momento de considerar tanto los acuerdos de representación como las empresas productoras; sin embargo, los acuerdos de representación se reconocen más por el mecanismo de competencia que por el de cooperación. La representación está encaminada a que el socio sea un instrumento para llegar al mercado meta, aunque es una estrategia para iniciar actividades de colaboración, que posteriormente abrirán paso a relaciones más complejas y permanentes.

De forma complementaria, se entrevistaron a 34 informantes clave de empresas mexicanas del sector ambiental participantes en Al-Invest (tabla 4). Se considera importante el análisis sobre México por ser una región estratégica para la cooperación triangular, y en todo caso, para las empresas europeas del sector ambiental que incursionan en América Latina. Lamentablemente, no todas las empresas estuvieron dispuestas a colaborar con el presente proyecto, de ahí que las entrevistas alcanzadas sean un esfuerzo exhaustivo en el que los límites en información dependen mucho del nivel de apertura y colaboración de los empresarios, así como de los recursos para la realización de visitas de campo.

Tabla 4. Aspectos de interés con base en 34 empresas mexicanas del sector ambiental participantes en Al-Invest

\begin{tabular}{|c|c|c|}
\hline Aspectos de interés & $\begin{array}{c}\text { Porcentaje de } \\
\text { empresas (con base en } \\
34 \text { entrevistas) }\end{array}$ & $\begin{array}{l}\text { Número de } \\
\text { empresas }\end{array}$ \\
\hline $\begin{array}{l}\text { Han enfrentado situaciones calificadas como fracaso } \\
\text { dentro de un vínculo empresarial. }\end{array}$ & $44 \%$ & 15 \\
\hline $\begin{array}{l}\text { Han aumentado su rentabilidad a través del vínculo } \\
\text { de cooperación internacional. }\end{array}$ & $79 \%$ & 27 \\
\hline Han establecido vínculos con empresas alemanas. & $14,7 \%$ & 5 \\
\hline $\begin{array}{l}\text { Han establecido vínculos con empresas } \\
\text { estadounidenses. }\end{array}$ & $20,58 \%$ & 7 \\
\hline $\begin{array}{l}\text { Han establecido vínculos con empresas europeas } \\
\text { (incluidas las alemanas). }\end{array}$ & $29.4 \%$ & 10 \\
\hline
\end{tabular}

Fuente: elaboración propia con base en los resultados alcanzados en los proyectos Generación de valor y cooperación internacional en las empresas de menor tamaño de Iberoamérica, UDG-CA-484. 
Las empresas mexicanas del sector ambiental han encontrado que los vínculos con empresas de otras regiones aumenta su rentabilidad. Algunas de ellas han enfrentado fracasos derivados de los vínculos emresariales; sin embrago, y a la manera de Axelrod (1984), continúan cooperando con agentes económicos de otras regiones, en especial de la Unión Europea, y más específicamente con Alemania, y Estados Unidos (tabla 4). A continuación se presentan brevemente algunos de los casos analizados. Los dos primeros se han beneficiado de más de un evento Al-Invest organizado por el Eurocentro Nafin, mientras que el tercer y último caso sólo asistieron una vez, pero han manetnido vínculos débiles con otras empresas, que les han permitido continuar sus actividades económicas. Los nombres de las empresas se conservan en anónimo, conforme a la confidencialidad solicitada por la Unión Europea a sus bases de datos. También se ha eliminado aquellos datos que permitan identificar fácilmente a las empresas como son nombres de contactos, las direcciones, nombres de empresas asociadas o marcas de productos. Sólo se consideran los hechos que permiten enriquecer el análisis de los resultados.

\subsection{Empresa "Gestión de aguas residuales"}

Esta empresa cuenta con ochenta y ocho empleados y se ubica en Ciudad de México. Tiene dieciocho años de existencia y mantiene vínculos con empresas de Holanda, Alemania, Estados Unidos, Canadá y Brasil. Se especializa en el diseño y construcción de plantas de tratamiento de agua residual, reutilización del agua y agua potable. Cuentan con tecnología de punta, bajos costos, alta eficiencia y calidad. Entre sus clientes seencuentran gobiernos, papeleras, cerveceras, productoras de lácteos, embotelladoras, textileras, farmacéuticas, procesadoras de pollo, sanitarias, mineras y destileras, entre otros. Algunos de sus clientes son empresas que operan globalmente.

\subsection{Empresa "Consultora en manejo del agua"}

Esta empresa cuenta con menos de cien empleados, veinte años de experiencia y tiene oficinas en tres ciudades de Mèxico. Ofrece soluciones específicas en el manejo y ahorro de agua, y en energía industrial. A principios de este siglo intentó vincularse a una empresa brasileña, pero esto se tradujo en un fracaso. De acuerdo 
a la entrevista, hubo conflicto de intereses, mala comunicación y la empresa brasileña comercializaba con la tecnología de otros. En 2008, el empresario expresó que, a pesar de los resultados, estaba dispuesto a buscar nuevos colaboradores en el extranjero. A partir de 2011 se unió a un vínculo multiempresa (González, 2008) conformado por Ecuador, Panamá, Colombia, Chile y China. El vínculo multiempresa que han construido mantiene, a su vez, vínculos con empresas europeas, asiáticas y norteamericanas. A través de los vínculos obtienen nuevos medios para el tratamiento del agua, anticipan las necesidades del mercado, amplían su know-how y logran integrar y desarrollar tecnologías más eficientes.

\subsection{Empresa "Manejo de residuos"}

Se considera una organización comprometida con las empresas que se preocupan por mejorar y controlar sus procesos y disponer de manera adecuada los residuos industriales y biológicos infecciosos. Mantiene vínculos débiles (Granovetter, 1973) con empresas de Alemania, España y Canadá. Tiene dieciocho años de antigüedad. Está conformada por treinta empleados. La mayor parte de sus actividades se realizan mediante la subcontratación y se consideran intermediarios. La principal razón es financiera, el sector ambiental requiere de grandes inversiones, lo que conduce a que las empresas mexicanas se vinculen con empresas extranjeras capaces de hacer frente a los costos implicados en cada operación y que cuenten con investigación y desarrollo, así como tecnología de punta para el diagnóstico y monitoreo sobre la gestión de residuos. Los servicios que ofrece son: trámites ambientales; recolección, almacenamiento, manejo de residuos; venta de contenedores, limpieza de fosas e impacto ambiental.

Las tres empresas presentadas en este espacio a discusión se consideran casos típicos porque en sus origenes son empresas ciento por ciento mexicanas, que han sabido adaptarse al sector ambiental y vencer las barreras tecnológicas y legales por medio de los vínculos con empresas de otras regiones.

\section{CONCLUSIONES}

El sector ambiental facilita la existencia de empresas de menor tamaño en el marco de la cooperación triangular y a través de las redes de cooperación empresarial internacionales. Este sector fomenta la cooperación Norte-Sur-Sur en el marco de la cooperación triangular para el desarrollo. El impacto en el desarrollo local por las empresas 
involucradas no está orientado hacia la creación de nuevos puestos de empleo, sino que se orienta hacia el uso de energías alternativas y el manejo de contaminantes. En otras palabras, busca la transformación de las actividades económicas. El presente estudio muestra un número reducido de empresas participantes, sin embargo, es posible que el monto de las inversiones y de los intercambios hacia dentro de la red sean considerables, dada la naturaleza del sector estudiado. Finalmente, es un sector atractivo para estudiar con mayor profundidad la transferencia de conocimiento Norte-Sur-Sur.

\section{REFERENCIAS}

Abdenur, E., \& de Souza, M. (2013). Cooperación china en América Latina. Las implicaciones de la asistencia para el desarrollo. Íconos-Revista de Ciencias Sociales, (47), 69-85.

Achkar, M. (2005). Indicadores de sustentabilidad. En M. Achkar, V. Cantón, \& R. Cayssials. Ordenamiento ambiental del territorio. (pp. 55-70). Montevideo: DIRAC.

Aguilera, F., Canter, L. W., Esser-Winckler, H., Jalal, K. F., Rogers, P. P., Koolen, R., ... Lusk, P. (1995). Economía de los recursos naturales: un enfoque institucional. Santiago de Chile: CEPAL.
Albizu, E., Olazaran, M., Otero, B., \& Lavía, C. (2011). Innovación en las pymes industriales: una visión desde el modelo interactivo. Revista Internacional de Organizaciones, (7), 17-43.

Alderete, M. (2007). Nuevas tecnologías de la información y de la comunicación: factores explicativos de la conducta exportadora en Argentina. Economía y sociedad, 12(20), 35-61.

Aray, A., Caraballo, L., \& Ruiz, M. (2002). Diagnóstico del impacto ambiental que ocasionan las empresas que operan en el Sector Cúcuta del Municipio Maturín: Un Caso de Responsabilidad Social (tesis de grado). Universidad de Oriente, Maturín.

Axelrod, R. (1984). The Evolution of Cooperation. Nueva York: Basic Books.

Banco Mundial (2012). Medio ambiente. Resultados del sector. Recuperado de http://www. bancomundial.org/es/results/ 2013/04/13/environment-results-profile

Banerjee, S. (2002). Corporate Environmentalism. The Construct and its Measurement. Journal of Business Review, (55), 177-191.

Barton, R. (1998). La dimensión norte-sur de las industrias de limpieza ambiental y la difusión de tecnologías límpias. Revista de la CEPAL, (64), 129-150. 
Bercovich, N., \& Chidiak, M. (1994). Reestructuración industrial y gestión ambiental en el sector de celulosa y papel en Argentina. Documentos de trabajo $\mathrm{N}^{\circ} 16$. Buenos Aires: Centro de Investigaciones para la Transformación. BMZ (2013). Cooperaciones triangulares en la cooperación alemana para el desarrollo. Berlín: Ministerio Federal de Cooperación Económica y Desarrollo (BMZ). Buenaventura, A., Paime, L., \& Villegas, G. (2006). Una aproximación a los perfiles ambientales de la empresa. Innovar, 16(28), 57-74. Bueno, C., \& Saraví, G. (1997). Relaciones de cooperación interempresariales. Lecciones de la experiencia mexicana. Nueva Sociedad, 9, 102-121.

Buil, I., Fraj., E., \& Matute, J. (2005). Tipología de empresas medioambientales en el sector de bienes de consumo final. Economía y Empresa. (XXIII), 54-55.

Camacho, M. (2016). La capacidad potencial de influencia del sector industrial manufacturero: Un análisis del Programa de Inspección y Vigilancia Industrial en México. Gestión y Política Pública, 1(XXV), 33-79.

Campos-Arce, J., Finegan, B., \& Villalobos-Soto, R. (2001). Manejo diversificado del bosque: aprovechamiento de bienes y servicios de la biodiversidad del bosque neotropical. Revista Forestal Centroamericana, (36), 6-13.

Campos, J., Alpízar, F., Louman, B., Parrotta, J., \& Madrigal, R. (2006). Enfoque integral para esquemas de pago por servicios ecosistémicos forestales. En G. Mery, R. Alfaro, M. Kanninen, M. Lobovikov, Forest in the Global Balance-Changing Paradigms. World Series Vol. 17, (pp. 97-116). Viena: IUFRO.

Canter, W., Dauner, I., Gómez, I., Ruiz, A., Lutz, E., Binswanger, P., ... Taylor, G. (1998). Manual de evaluación de impacto ambiental: técnicas para la elaboración de estudios de impacto. Washington: Banco Mundial.

Capó-Vicedo, J., Miquel, T., \& Expósito-Langa, M. (2009). Redes virtuales de PYMES. Un caso de estudio en el sector textil español. Dirección y organización, (38), 66-77.

Castiblanco, C. (2003). Alcances y limitaciones de la valoración económica de los bienes y servicios ambientales. Revista Ensayos de Economía, 13, 1-2.

Castro, L. F. (2002). Aplicación del principio contaminador-pagador en América Latina: evaluación de la efectividad ambiental y eficiencia económica de la tasa por contaminación hídrica en el sector industrial colombiano. Santiago de Chile: Naciones Unidas. 
CEPAL (2014). La inversión extranjera directa en América Latina y El Caribe. Santiago de Chile: CEPAL.

Cervilla, M., \& Lorenzo, O. (1999). Redes de empresas y tecnologías de información: Opciones para el desarrollo de la PYME. Debates IESA, 5(1), 230-248.

Del Brío, J., \& Junquera, B. (2001). Factores inductores del comportamiento medioambiental en las empresas industriales españolas. Economía y Empresa, 41(XV), 75-96.

Donovan, J., Stoian, D., \& Junkin, R. (2004). Cadena de valor como estrategia para el desarrollo de eco-PyME en América Tropical. Memorias de la Semana Científica, 17-18.

Falconí, F., \& Burbano, R. (2004). Instrumentos económicos para la gestión ambiental: decisiones monocriteriales versus decisiones multicriteriales. Revista de la Red Iberoamericana de Economia Ecológica, 1, 11-20.

Fernández, G., \& Narváez, M. (2011). Asociatividad empresarial: estrategia para la competitividad en las pymes del sector turístico. Visión Gerencial, 10(2), 295-308.

Fernández, Z., \& Revilla, A. (2010). Hacer de la necesidad virtud: los recursos de las pymes. Economía industrial, 375, 53-64.
Fernández-Jardón, C. M. (2012). Determinantes de la capacidad de innovación en PYMES regionales. Revista de Administração da UFSM, 5, 749-765.

Ferraro, C. A., \& Stumpo, G. (2010). Políticas de apoyo a las PYME en América Latina entre avances innovadores y desafios institucionales. Santiago de Chile: Naciones Unidas.

Fonseca, S. A. (2006). El café de sombra: un ejemplo de pago de servicios ambientales para proteger la biodiversidad. Gaceta ecológica, (80), 19-31.

Flyvbjerg, B. (2006). Five Misunderstandings about Case-Study Research. Qualitative inquiry, 12(2), 219-245.

Fujita, M., Krugman, P., \& Venables, A. (2000). Economía espacial: las ciudades, las regiones y el comercio internacional. Madrid: Ariel.

Fundación Entorno (2003). Informe sobre la Gestión Ambiental en la Empresa Española. Avances hacia la Sostenibilidad. Madrid: Fundación Entorno.

García, M. B., \& Moreno, F. A. (2007). Estrategias de internacionalización de la PYME española: una visión desde el programa de apoyo a proyectos de inversión (papi). Información Comercial Española, ICE: Revista de economía, (839), 55-64. 
German Agency for Technical Cooperation (2005). Identificación de áreas de oportunidad en el sector ambiental de América Latina y el Caribe. Casos exitosos de colaboración entre industrias para formular alianzas. Santiago de Chile: CEPAL.

Giménez, G.; Casadesús, M., \& Valls, J. (2002). Gestión ambiental y competitividad: situación actual en la industria española. ESIC Market, (112), 211-224.

Gómez, M.; Ayllón, B., \& Albarrán, M. (2011). Reflexiones prácticas sobre cooperación triangular. Madrid: CIDEAL.

González, T. (2003). Las redes de cooperación empresarial internacionales. Un camino hacia el mercado asiático para la PYME mexicana. En Memoria del VIII Congreso Internacional en Contaduría Administración e Informática. México: Universidad Nacional Autónoma de México.

González, T. (2007). Redes de cooperación empresarial internacionales vs redes locales. Revista Venezolana de Gerencia, 12(37), 9-26.

González, T. (2008). Las ventajas y desventajas que el vínculo multiempresa y la red de cooperación empresarial internacional presentan para la PYME. Redes, Revista Hispana para el análisis de redes sociales, (14), 1-26.
Granovetter, M. (1973). The Strength of Weaks Ties. American Journal of Sociology, Vol(Núm), 1360-1380.

Hernández, R. (2001). Elementos de competitividad sistémica de las pequeñas y medianas empresas (PYME) del Istmo Centroamericano. Santiago de Chile: Naciones Unidas.

Kulfas, M. (2009). Las PYMES argentinas en el escenario post convertibilidad: políticas públicas, situación y perspectivas. Documentos de proyectos, N. 40, Santiago de Chile: Naciones Unidas.

Lafer, C. (1973). Comercio Internacional: Fórmulas jurídicas y realidades político-económicas. Foro Internacional, 14(2 (54), 204-219.

Leonard, J., Cox, M., Chateauneuf, R., Cirio, F., Otero, M., Mellor, J., ... Pomareda Benel, F. (1987). Recursos naturales y desarrollo económico en América Central: Un perfil ambiental regional. Turrialba: CATIE.

Liendo, M., \& Martínez, A. (2001). Asociatividad. Una alternativa para el desarrollo y crecimiento de las PYMES. En Memoria de las Sextas Jornadas de Investigaciones en la Facultad de Ciencias Económicas y Estadisticas. Rosario: Universidad Nacional del Rosario. 
Lomas, L., Martín, B., Louit, C., Montoya, D., Montes, C., \& Álvarez, S. (2005). Guía práctica para la valoración económica de los bienes y servicios ambientales de los ecosistemas. España: Fundación Interuniversitaria Fernanda González Bernáldez.

Luna, R. (2009). PYMES y cadenas de valor globales. Implicaciones para la política industrial en las economías en desarrollo. Análisis Económico, 24(57), 199-216.

Martínez, J., Geddes, P., Podolinky, A., Castro, L., Aguilera, F., Trejos, R., ... Barril, A. (1995). Los principios de la economía ecológica. San José: Universidad de Costa Rica.

Moncayo, F. (2010). Asociatividad y Cadenas de Valor en Ecuador. Revista de la Universidad del Azuay, (51), 81-97.

Ortiz, A., Izquierdo, H., \& Rodríguez, C. (2013). Gestión ambiental en PYMES industriales. Interciencia, (38), 179-185.

Panwar, R., \& Hansen, E. (2008). La responsabilidad social de la empresa en el sector forestal. Unasylva: Revista Internacional de Silvicultura e Industrias Forestales, (230), 45-48.

Pascual, U., \& Corbera, E. (2011). Pagos por servicios ambientales: perspectivas y experiencias innovadoras para la conservación de la naturaleza y el desarrollo rural. Revista Española de Estudios Agrosociales y Pesqueros, (228), 11-29.

Penna, A., \& Cristeche, E. (2008). La valoración de servicios ambientales: diferentes paradigmas. Estudios socioeconómicos de la sustentabilidad de los sistemas de producción y recursos naturales, (2) 1-44.

Perego, H., \& Marteau, S. (2007). Integración de cadenas productivas de valor. Contribuciones a la economía, 28, 1-14.

Pla-Barber, J., \& Escribá, A. (2010). La influencia de las características y percepciones directivas en los nuevos modelos de internacionalización. Una aplicación en la pyme manufacturera tradicional. Economía industrial, (375), 101-112.

Porter, G., Brown, W., Meadows, H., Meadows, L., Randers, J., Gosselin, S., \& Kussow, W. (1991). Global Environmental Politics. Gland: IUCN.

Ruzo, E., Losada, F., Díez, J., \& Barreiro, B. (2005). Estrategia medioambiental de la empresa exportadora gallega: Ventajas e inconvenientes percibidos. Proceedings of the XV Spanish-Portuguese Meeting of Scientific Management, 389-403.

Sandrea, M., \& Boscán, M. (2010). Gerencia ambiental en el sector 
zuliano de manufacturas plásticas. Espacio Abierto, 19(3), 555-571. Schaper, M. (2007). Los desafíos del desarrollo sostenible en las economías abiertas de América Latina y el Caribe. Pensamiento Iberoamericano, 1, 199-213.

Tabares, S., Anzo, E., \& Estrada, M. (2014). La cooperación internacional en la internacionalización de pequeñas y medianas empresas de Medellín: un estudio de caso comparado. Estudios Gerenciales, 30(132), 314-324.

Tkachuk, C. (2004). Innovación y Territorio como factores de entorno para la competitividad de las PYMES. Hacia nuevas estrategias del desarrollo local. Documento de trabajo. Universidad Nacional de Quilmes. Recuperado de http://hm.unq.edu.ar/archivos_hm/CT_innovacion_territorio.pdf

Torres-Carral, G. (2006). El pago de los servicios ambientales y las comunidades indígenas. $R a$ Ximhai, 2(1), 187-207.

Ueki, Y., Tsuji, M., \& Cárcamo, R. (2005). Tecnologías de la información y las comunicaciones
(TIC) para el fomento de las PYMES exportadoras en América Latina y Asia Oriental. Santiago de Chile: CEPAL.

Vargas, E., Zizumbo, L., Viesca, C., \& Del Carmen Serrano, R. (2011). Gestión ambiental en el sector turístico mexicano. Efectos de la regulación en el desempeño hotelero. Cuadernos de Administración, 24(42), 183-204.

Velásquez, F. (2004). La estrategia, la estructura y las formas de asociación: fuentes de ventaja competitiva para las pymes colombianas. Estudios gerenciales, 20(93), 73-97.

Villarreal, M. (2006). Bienes y servicios ambientales en México: caracterización preliminar $y$ sinergias entre protección ambiental, desarrollo del mercado $y$ estrategia comercial Vol. 119. Nueva York: Naciones Unidas. Yoguel, G., \& Bercovich, N. (1994). Cooperación empresarial en la que intervienen firmas brasileñas y argentinas: primeras reflexiones que surgen del trabajo de campo. Buenos Aires: CEPAL. 